\title{
Effects of a task-specific warm-up on anaerobic
} power

\author{
J.A. Hawley, MA, M.M. Williams, MSc, G.C. Hamling, MNZSP and R.M. Walsh, MNZSP \\ School of Physiotherapy, Faculty of Health Studies, Auckland Technical Institute, New Zealand
}

Twenty-four untrained (UT) males (age $21 \pm 2.5 \mathrm{yr}$, height $1.77 \pm 0.05 \mathrm{~m}$, weight $75.3 \pm 10.1 \mathrm{~kg}$, values mean $\pm S D$ ) performed the Wingate Anaerobic Test (WT) under two conditions, cold (C) and following a warm-up (WU). Trials were separated by a minimum of $48 \mathrm{~h}$. A modified Monark 818 cycle ergometer was interfaced with an Apple IIE microcomputer and peak power (PEAK), mean power (MEAN) and fatigue index (FI) determined. The WU trial consisited of an $8 \mathrm{~min}$ incremental continuous cycling bout (cadence 90 rev. $\mathrm{min}^{-1}$ ) with $5 \mathrm{~min}$ rest before the WT. During the $C$ trial subjects completed only the WT. A repeated measures design was employed with order of trials counterbalanced.

ANOVA revealed no significant differences for PEAK or MEAN between WU or C conditions. However FI was significantly greater $(p<0.05)$ following the WU. A significant correlation $(r=0.45, p=0.03)$ was obtained between WU intensity and FI.

These findings suggest that our UT subjects were fatiguing themselves during the WU. Future studies are needed to assess whether a task-specific WU in which FI is not impaired would lead to improvements in PEAK and MEAN. Investigators should be aware that a self-spaced WU may increase FI in the WT in UT subjects.

Keywords: Anaerobic power, warm-up, Wingate test

\section{Introduction}

Warm-up (WU) exercise prior to strenuous physical activity is a generally accepted practice among athletes. Indeed, most athletes incorporate some form of preliminary activity or WU procedure into their training routines, especially if they anticipate a strenuous workout ${ }^{\prime}$. Although WU is a simple ergogenic aid, its efficacy is essentially unsubstantiated.

Improved performance resulting from WU exercise has been reported in a number of studies ${ }^{2}$. Other investigations however demonstrate no such effects ${ }^{7-9}$. To date, few experiments have dealt with the effects of WU on high intensity supramaximal exercise which leads to exhaustion in less than one minute.

In recent years, the Wingate Anaerobic Test (WT) has been employed by a growing number of laboratories for evaluating the capacity for short-term exhaustive work ${ }^{4,10-12}$. To date, only one study has assessed the effects of WU on performance in the $\mathrm{WT}^{4}$.

Address for correspondence: John A. Hawley, School of Physiotherapy, Faculty of Health Studies, Auckland Technical Institute, Auckland, New Zealand

(C) 1989 Butterworth \& Co (Publishers) Ltd 0306-3674/89/040233-04\$03.00
In that investigation, a 15 min intermittent ( $30 \mathrm{~s}$ on, $30 \mathrm{~s}$ off) treadmill WU improved mean power by seven per cent $(p<0.005)$ in a group of young untrained (UT) males ignorant of the concept of WU. The nature of the WU however was not task specific.

Other studies have examined the effects of WU on maximal performance tasks other than the $\mathrm{WT}^{3,13}$. Genovely and Stamford compared the effects of no WU with prolonged WU (60 min) at 40 per cent and 68 per cent of $\mathrm{VO}_{2}$ max on bouts of maximal work on a cycle ergometer. Their lower intensity WU did not alter performance, while the higher intensity WU impaired performance relative to no WU. It is unclear however whether these findings can be generalized to WU of shorter duration. The purpose of this investigation was to assess the effect of a short duration taskspecific incremental WU in UT subjects in the WT.

\section{Methods \\ Subjects and experimental protocol}

Twenty-four UT male students volunteered to participate in this investigation. Prior to their first visit to the laboratory, subjects were advised as to the nature of the experimental protocol but were not informed of the specific purpose of the study. The subjects were led to believe that the study concerned gathering normative data on the WT using two differing protocols. Subjects signed an informed consent form in accordance with the guidelines outlined by the American College of Sports Medicine ${ }^{1 t}$. The experimental protocol was approved by the department's ethics committee.

A mechanically-braked Monark 818 cycle ergometer (Watson Victor, Auckland, NZ) was calibrated as previously described ${ }^{15}$. It was modified with toe clips, straps, adjustable racing handlebars and racing saddle, and interfaced with an Apple IIE microcomputer. An Optron OPB 125A (TRW, Optron, Auckland, NZ) reflective object sensor was mounted parallel to the ergometer flywheel, and a series of 36 (10 degree intervals) reflective to non-reflective transitions marked on the flywheel. Sample periods of 0.5 s enabled calculation of average flywheel frequency which was subsequently scaled to pedal frequency.

The WT consists of a 30 s exhaustive cycling exercise bout against a resistance determined by the bodyweight and training status of the individual. The validity and reliability of this test have been documented 
Effects of task-specific warm-up on anaerobic power: J.A. Hawley et al.

Table 1. Power output during the warm-up

\begin{tabular}{lr}
\hline $\begin{array}{c}\text { Time period } \\
\text { (min) }\end{array}$ & $\begin{array}{c}\text { Power } \\
(W)\end{array}$ \\
\hline $0-5$ & $79.9( \pm 46.3)$ \\
$5-7$ & $190.9( \pm 69.5)$ \\
$7-8$ & $296.3( \pm 82.7)$ \\
\hline
\end{tabular}

Values are mean $\pm \mathrm{SD}$

previously ${ }^{16,17}$. Three parameters are determined from the WT: the peak power (PEAK) elicited during the test, taken as the highest average power for any $5 \mathrm{~s}$ period; the mean power (MEAN) that is sustained throughout the $30 \mathrm{~s}$, and the fatigue index (FI) or power drop-off during the test. Subjects reported to the laboratory a minimum of $3 \mathrm{~h}$ post-absorptive and received standardized written instructions as to the testing procedure.

All subjects performed under the two separate test conditions; cold (C) and following a WU. Saddle height and handlebar position were the same for both tests. Order of trials was counterbalanced. Testing was carried out between $1200-1600 \mathrm{~h}$ with a minimum of $48 \mathrm{~h}$ separating trials.

During the WU trial, subjects performed a continuous $8 \mathrm{~min}$ incremental exercise bout on a cycle ergometer (cadence $90 \mathrm{rev} \cdot \mathrm{min}^{-1}$ ). For the first $5 \mathrm{~min}$ subjects were asked to cycle at a workload they perceived as 'very, very light'. For the next 2 min they were asked to cycle at a workload they perceived to be 'moderate', and for the final minute at a workload they perceived as 'moderately heavy'.

Subjects were shown how to adjust the resistance settings on the ergometer and were free to alter the workload at will throughout the WU. Upon completion of the WU, subjects dismounted the ergometer and were seated for $4 \mathrm{~min}$.

After this rest period, subjects remounted the ergometer and the feet were firmly strapped. Five minutes after the completion of the $\mathrm{WU}$, subjects began pedalling at minimal resistance and gradually increased the cadence to 115-120 rev. $\mathrm{min}^{-1}$.

When this cadence was attained, the full load $(0.075$ $\mathrm{kp} . \mathrm{kg}^{-1}$ bodyweight) was manually applied by an investigator and the computer activated. Subjects were instructed to remain seated and verbally encouraged to maintain maximal pedal rates throughout the test. The time taken to load the subjects was in the order of $1.0-1.5 \mathrm{~s}$.
Although recent reports ${ }^{10,18}$ have shown the optimal force to be higher than originally recommended by the Wingate group, the issue of optimal force settings in the WT has not been fully resolved ${ }^{17}$. The choice of load setting in this investigation was based on a pilot study of UT subjects. Further, a similar load has been used in other laboratories ${ }^{19-21}$ and permits comparisons with available literature.

During the $C$ trial subjects were given no WU but were required to perform the WT as previously described.

Upon completion of their second trial, all subjects completed a post-study questionnaire to ascertain their perception of the purpose of the study.

\section{Statistical analysis}

Data was analysed by a repeated measures analysis of variance (ANOVA) and Pearson Product moment correlations, using the statistical software programme SYSTAT (SYSTAT Inc., Evanston, IL). Results were considered significant where $p<0.05$.

\section{Results}

The mean $( \pm S D)$ age $(\mathrm{yr})$, height $(\mathrm{m})$ and weight $(\mathrm{kg})$ of our subjects was $21.0( \pm 2.5), 1.77( \pm 0.05), 75.3$ $( \pm 10.1)$. Table 1 displays the mean power outputs attained during the WU prior to the WT. The mean power output during the entire eight minutes was 134.7 $\mathrm{W}( \pm 59.6)$ and $226.0 \mathrm{~W}$ over the last three minutes.

Table 2 displays the performance data for the WT under the two trial conditions. No significant differences were found for PEAK or MEAN between the WU and C trials. However, FI was significantly greater $(p<0.05)$ following the WU trial.

Results from the post-study questionnaire revealed that no subject perceived the purpose of the study to be concerned with assessing the effects of a WU on performance.

\section{Discussion}

PEAK and MEAN power outputs obtained during the WT in this study are in the order of those previously reported for UT individuals ${ }^{17,21}$. There were no significant differences in either of these variables under the two trial conditions as shown in Table 2. The FI however, was significantly greater $(p<0.05)$ following the WU.

Table 2. Performance data for the Wingate Anaerobic Test

\begin{tabular}{|c|c|c|c|c|c|}
\hline Trial & $(W)$ & $\left(W \cdot k g^{-1}\right)$ & $(W)$ & $\left(W \cdot k^{-1}\right)$ & $\begin{array}{c}\text { Fatigue } \\
\text { index } \\
(\%)\end{array}$ \\
\hline $\begin{array}{l}\text { Cold } \\
\text { Warm }\end{array}$ & $\begin{array}{c}855.4 \\
( \pm 81.2) \\
867.1\end{array}$ & $\begin{array}{c}11.5 \\
( \pm 1.2) \\
11.6 \\
(+14)\end{array}$ & $\begin{array}{r}655.5 \\
( \pm 66.4) \\
654.5 \\
(+908)\end{array}$ & $\begin{array}{r}8.8 \\
( \pm 0.9) \\
8.7 \\
(+12)\end{array}$ & $\begin{array}{c}41.9 \\
( \pm 6.8) \\
44.1^{*} \\
(+78)\end{array}$ \\
\hline
\end{tabular}

Values are mean $\pm S D$

*Indicates significantly greater than $C$ trial $(p<0.05)$

Fatigue Index $=((\mathrm{PEAK}-\overline{\mathrm{X}}$ FINAL $5 \mathrm{~s}) / \mathrm{PEAK}) \times 100$ 
Effects of task-specific warm-up on anaerobic power: J.A. Hawley et al.

These results demonstrate that our task-specific incremental WU did not improve performance in the WT in our UT individuals. This finding contrasts with a previous investigation ${ }^{4}$ which reported a highly significant ( $\mathrm{p}<0.005$ ) improvement in MEAN in the WT in young males who undertook a 15 minute intermittent treadmill WU at approximately 60 per cent of $\mathrm{VO}_{2}$ max.

The disparity between the results of the current investigation and that of Inbar and $\mathrm{Bar}-\mathrm{Or}^{4}$ can probably be explained by the different nature of the two WU protocols. The WU used in this present study was a continuous incremental task which, in our UT subjects, probably had a fatiguing effect which subsequently impaired performance in the WT. This is supported by the finding that the FI was significantly higher $(p<0.05)$ in subjects following a WU.

Post-hoc analyses revealed two other findings in this regard. First, the intensity with which subjects undertook their WU was not significantly related to their performance in the WT. Pearson correlation coefficients between mean power during the WU and PEAK and MEAN during the WT were -0.01 and -0.27 respectively. This indicates that the intensity with which subjects were undertaking the WU was unrelated to their individual physical capacity and subsequent performance in the WT. Second, there was a significant correlation $(\mathrm{r}=0.45, \mathrm{p}=0.028)$ between the mean WU intensity and the subsequent FI during the WT. This suggests that MEAN was impaired for some subjects due to an excessively vigorous WU.

Although oxygen uptake was not directly assessed during our WU, if we assume the normal active 25 year old male has a $\mathrm{VO}_{2} \mathrm{max}$ of around $40 \mathrm{ml} \cdot \mathrm{kg} \cdot \mathrm{min}^{-1} 22$ estimations of $\mathrm{VO}_{2}$ from a predictive equation ${ }^{23}$ would indicate that our UT subjects were performing at approximately 88 per cent of $\mathrm{VO}_{2}$ max during the penultimate two minutes of the WU, and in excess of their aerobic power during the final minute of the WU.

Results of a recent investigation ${ }^{24}$ suggest that lack of glycogen may be an important factor in the development of fatigue during exercise at intensities higher than $\sim 90$ per cent of $\mathrm{VO}_{2}$ max. Further, while glycogen is broken down mostly in type I fibres during exercise of low intensity (43 per cent of $\mathrm{VO}_{2}$ max), high intensity exercise (91 per cent $\mathrm{VO}_{\text {max }}$ ) results in glycogen breakdown in all fibre types ${ }^{24}$. It has also been established that type I, IIA and IIB fibres are activated during the first four to seven minutes of exercise at intensities greater than 84 per cent of $\mathrm{VO}_{2} \mathrm{max}^{25,26}$. These findings suggest that in our UT subjects, glycogen depletion would have occurred during the WU, which may have resulted in the subsequent performance reduction in the WT.

While our study was unable to demonstrate any difference in performance indices of the WT following a $W U$, further investigations are needed in this area. Our UT subjects were unable to pace themselves effectively on our continuous incremental WU protocol. Future studies are needed to identify more appropriate self-pacing instructions such that subjects will not excessively fatigue themselves during a WU.

Alternatively, if a self-pacing protocol is not employed, the intensity and duration of the WU must be individualized to the particular fitness level of the subject and the anticipated physical task. Invest- igators should be aware that UT subjects may impair performance in the WT if a WU is too intense.

\section{Acknowledgements}

The authors wish to thank Phillip J. Handcock and Michael Vickovic for technical assistance. This study was supported by a grant from Fitness Concepts (NZ) Litd.

\section{References}

1 Shellock, F.G. Physiological benefits of warm-up Phys Sportsmed 1983, 11, (10), 134-139

2 Asmussen, E. and Boje, O. Body temperature and capacity for work Acta Physiol Scand 1960, 48, 448-453

3 De-Bryun-Prevost, $P$. and Lefebvre, F. The effects of various warming up intensities and durations during a short maximal anaerobic exercise Eur I Appl Physiol 1980, 43, 101-107

4 Inbar, O. and Bar-Or, O. The effects of intermittent warm-up in 7-9 year old boys Eur J Appl Physiol 1975, 34, 81-89

5 Ingjer, F. and Stromme, S.B. Effects of active, passive or no warm-up on the physiological response to heavy exercise Eur J Appl Physiol 1979, 40, 273-282

6 Martin, B, Robinson, S., Weigman, D. and Aulick, L. Effects of warm-up on the metabolic response to strenuous exercise Med Sci Sports 1975, 7, 146-149

7 Knowlton, R.G., Miles, D.S. and Sawka, M.N. Metabolic response of untrained individuals to warmup Eur J Appl Physiol 1978, 40, 1-5

8 Sedgewick, A.W. Effect of actively increased muscle temperature in local muscular endurance Res Quart $1964,35,532-538$

9 Sedgewick, A.W. and Whalen, H.R. Effect of passive warm-up on muscular strength and endurance Res Quart 1964, 35, 45-49

10 Evans, J.A. and Quinney, H.A. Determination of resistance settings for anaerobic testing Can J Appl Sport Sci $1981,6,53-56$

11 Jacobs, I. The effects of thermal dehydration on performance of the Wingate Anaerobic Test Int I Sports Med 1980, 1, 21-24

12 Taunton, J.E., Maron, H. and Wilkinson, J.G. Anaerobic performance in middle and long-distance runners Can I Appl Sport Sci 1981, 6, 109-113

13 Genovely, H. and Stamford, B.A. Effects of prolonged warm-up exercise above and below anaerobic threshold on maximal performance Eur J Appl Physiol $1982,48,323-330$

14 American College of Sports Medicine Policy statement regarding the use of human subjects and informed consent 1988, 20, (1), v

15 Astrand, P.O. 'Work tests with the bicycle ergometer' 1975, Monark-Crescent AB, Varberg, Sweden

16 Bar-Or, O., Dotan, R. and Inbar, O. A 30-second allout ergometric test - its reliability and validity for anaerobic capacity Israel J Med Sci 1977, 13, 326

17 Bar-Or, O. The Wingate anaerobic test. An update on methodology, reliability and validity Sports Med 1987, 4381-394

18 Dotan, R. and Bar-Or, O. Load optimization for the wingate anaerobic test Eur J Appl Physiol 1983, 51, 409_ 417

19 Inbar, O., Kaiser, P. and Tesch, P. Relationship between leg muscle fiber type distribution and leg exercise performance Int J Sports Med 1981, 2, 154-159

20 Jacobs, I., Bar-Or, O., Karlsson, J., Dotan, R., Tesch, P., Kaiser, P. and Inbar, O. Changes in muscle metabolites in females with 30-s exhaustive exercise Med Sci Sports Exerc 1982, 14(6), 457-460 
Effects of task-specific warm-up on anaerobic power: J.A. Hawley et al.

21 Patton, J.F. and Duggan, A. Upper and lower body anaerobic power: Comparison between biathletes and control subjects Int I Sports Med 1987, 8, 94-98

22 Costill, D.L. A scientific approach to distance running Track and field news, 1979

23 American College of Sports Medicine, 'Guidelines for graded exercise testing and exercise prescription' 1986, Lea \& Febiger, Philadelphia

24 Vollestad, N.K. and Blom, P.C.S. Effect of varying exercise intensity on glycogen depletion in human muscle fibres Acta Physiol Scand 1985, 125, 395-405

25 Andersen, P. and Sjogaard, G. Selective glycogen depletion in the subgroups of type II muscle fibres during submaximal exercise in man Acta Physiol Scand 1976, 96, 26A

26 Essen, B. Glycogen depletion of different fibre types in human skeletal muscle during intermittent and continuous exercise Acta Physiol Scand 1978, 103, 446-455 